\title{
Online and On-demand Partitioning of Streaming Graphs
}

\author{
Ioanna Filippidou \\ Athens University of Economics and Business \\ 76 Patission Street, Athens, Greece \\ filippidoui@aueb.gr
}

\author{
Yannis Kotidis \\ Athens University of Economics and Business \\ 76 Patission Street, Athens, Greece \\ kotidis@aueb.gr
}

\begin{abstract}
Many applications generate data that naturally leads to a graph representation for its modeling and analysis. A common approach to address the size and complexity of these graphs is to split them across a number of partitions, in a way that computations on them can be performed mostly locally and in parallel in the resulting partitions. In this work, we present a framework that enables partitioning of evolving graphs whose elements (nodes and edges) are streamed in an arbitrary order. At a core of our techniques lies a Condensed Spanning Tree (CST) structure that summarizes the graph stream and permits computation of high-quality graph partitions both online and on-demand, without the need to ever look at the whole graph. The partitioning algorithm we present manages to create partitions from streaming graphs with low memory usage, but can also adapt partitions overtime based on different application needs such as minimizing cross-partition edges, balancing load across partitions, elastically adapting partitions based on a maximum load threshold and reducing migration cost. Our experiments with many different real and synthetic graphs demonstrate that our techniques manage to process and partition efficiently millions of graph nodes per second and also adapt them based on different requirements using only the information kept in the compressed CST structure, which can reduce the input graph size down to $1.6 \%$.
\end{abstract}

\section{INTRODUCTION}

In many applications voluminous data is naturally represented as a graph, where nodes model the entities and edges the complex interactions between them. Despite the size and the complexity of these graphs, it is still necessary to efficiently store, query and perform different kind of computations over their data. A prevalent approach is to split these graphs evenly across a number of partitions, in a way that all computations can be performed locally by different machines in parallel [9]. This problem is called balanced graph partitioning and the goal is to minimize the number of cross partition edges (edge-cut), while keeping the number of nodes in every partition approximately even.

Partitioning algorithms can be divided into two main classes. The first, termed static partitioning (offline), requires as input the whole graph in order to provide a solution. In cases where the data changes over time the procedure has to be performed from the beginning. In contrast, onepass (online) algorithms, assume that graph nodes are loaded continuously in a stream, each with its adjacency list, and the decisions of their partition placement is performed on the fly in order to incur as little computational overhead as possible. Online approaches are mostly used in cases when a graph does not fit in one machine and, thus, no global partitioning computations can be performed by an partitioner.

An often neglected aspect of graph partitioning is the management of changes as the graph evolves. Today data are becoming increasingly time-sensitive and in several applications graphs are dynamic by nature and require near realtime response to graph changes. For instance, the Twitter graph may receive thousands of updates per second at peak rate. As graph partitioning is an integral procedure of most computations needed over the data, it is critical to have the ability to keep up with the graph updates, by monitoring and adapting partitions over time. An initial partitioning scheme that doesn't adapt to changes, in dynamic graphs, may become ineffective quite fast. Static graph partitioning methods overlook this dynamic nature of real graphs. Even online approaches require a new stream of the whole graph in order to effectively decide the placement of the nodes since the locality (neighborhood information) may have changed from the previous stream.

In this paper we first define a fully streaming graph partitioning model that better addresses the requirements of modern applications. This model makes no assumptions of the way nodes and edges of a graph are streamed (unlike the online algorithms) and doesn't require any global information from the graph to be known in advance, as in the offline case. Nodes and edges of a graph are continuously streamed, as they are created by an application, and are processed in real-time by our techniques in order to be placed in a suitable partition. Unlike one-pass models, our approach is not restricted only to partitioning a static graph loaded in a stream, but is able to continuously process a dynamically changing graph.

Another main characteristic of our approach is the adaptive nature of its partitioning solutions. While the stream of the graph is processed by our techniques and at any point we can evaluate the quality of the current solution and calculate on the fly a new one with better balance or edge-cut quality. This is possible via the use of a summary structure, the Condensed Spanning Tree (CST) our algorithm maintains over the graph stream. Using this CST structure, partitions can be adapted as the graph evolves. Moreover, as the graph size changes, our approach can guarantee the elasticity of the system, by calculating solutions where the number of partitions $(k)$ changes over time. This way, the partitioning scheme can be adapted to better meet the demands of 
applications, making better use of the available resources.

Our contributions can be summarized as follows:

- We first propose a fully streaming graph partitioning scenario, where changes on the the graph are continuously processed by the system. Unlike prior techniques (offline and online), we do not assume that the full graph is available to the application nor a specific order of visiting the graph nodes and their edges.

- We provide an online node placement heuristic for the unrestricted streaming scenario that decides on the fly where to place incoming nodes in the current formed partitions, while maintaining the balance and the edgecut quality.

- We describe a flexible framework where applications can perform on demand partitioning of the graph at any time, adapting the partitions if necessary. Our techniques provide bounds for the resulting edge-cut of the computed partitions, enabling the applications to better manage the available resources. All necessary statistics for evaluating and adapting partitions are computed via a compact summary structure (CST) that is updated while processing the graph stream.

- We provide an extensive experimental evaluation using a diverse collection of synthetic and real-world graphs with various sizes and different edge distributions. Our experiments indicate that our techniques outperform existing algorithms, can quickly compute new partitions on demand and are able to process millions of streamed graph edges per seconds, using commodity hardware.

\section{PRoblem DEFinition}

A dynamic graph $G(t)=(V(t), E(t))$ is a graph whose vertices $V$ and edges $E$ change over time $t$, by addition of nodes and edges. The graph can be either directed or undirected, unweighted or weighted on both nodes and edges. While our techniques are generic, in what follows for ease of presentation, we assume the case of an undirected graph with weights on edges.

Graph partitioning objectives are common both for static and online partitioning and seek to divide the nodes of the graph into $k$ disjoint groups (partitions), where $V=\bigcup_{i=1}^{k} V_{i}$ and $V_{i} \cap V_{j}=\emptyset, \forall i \neq j$ with the following requirements:

Balance Criterion: Demands that all $k$ partitions have about equal size (or sum of node weights). It requires that, $\forall i \in\{1, \ldots, k\}\left|V_{i}\right| \leq(1+\epsilon)\lceil|V| / k\rceil$ for some imbalance parameter $\epsilon \geq 0$. In the case of $\epsilon=0$, the term perfectly balanced is used.

Edge-cut Minimization: A node $v$ is a neighbor of node $u$ if there exists an edge $(u, v) \in E$. If a node $v \in V_{i}$ has a neighbor $u \in V_{j}$, for $i \neq j$ then it is called boundary node. An edge that runs between partitions is also called cut edge. We refer to the number (or compound weight) of cut edges between two subsets $V_{a}$ and $V_{b}$ as the edge-cut between these two sets:

$$
\operatorname{edgecut}\left(V_{a}, V_{b}\right)=\sum_{u \in V_{a}} \sum_{v \in V_{b}} w(u, v)
$$

Let $\mathcal{P}=\left\{V_{1}, \ldots, V_{K}\right\}$ be a partitioning scheme of $V$ into $k$ parts. The objective function of our partitioner aims to minimize the sum of the weights of the edges between parts of $\mathcal{P}$, while respecting the balance criterion.

Although, objectives for partitioning are the same for static and online algorithms the manner that edges and nodes of the graph are processed differs in each category. Online solutions assume streams of nodes with adjacency lists, while static algorithms perform computations over the whole graph structure. In this work we consider a generic steaming model, termed "unrestricted streaming model" where new edges of the graph in the form $((u, v), w(u, v))$, where $u$ is the starting node, $v$ is the ending node and $w(u, v)$ the edge weight, are presented sequentially in a stream. The constituent nodes $u$ and $v$ that define the edge, may have already appeared earlier in the stream or, in case this is the first occurrence of one or both of them, observation of the edge implies their insertion in $V(t)$. Our model also handles applications where weights of existing edges and nodes are updated by the stream but we omit such examples for clarity.

The stream of graph edges is used in two different partitioning procedures presented in this work named online and on demand partitioning, respectively. In the "online partitioning” procedure, when new nodes are first presented in an edge stream, they take a partition assignment as soon as they arrive by using an online heuristic described in Section IV-A. On the other hand, in the "on-demand partitioning" scenario, the graph stream continuously updates a summary structure (the CST) that our technique maintains. Based solely on the CST information the partitioning monitor enables re-partitioning of nodes depending on the application needs, as described in Section IV-B. The framework is generic and permits execution of different strategies for adapting the partitions in order to consider the graph changes that occur over time.

\section{CONDEnsEd SPANnING TREE}

\section{A. Intuition and Definition of the CST}

The CST summary structure that is proposed and explained in this section, is a spanning tree of the graph that is formed gradually over the graph stream. In our work, we extend the spanning tree structure with additional statistics that are used to estimate the weight and the edge-cut of the proposed partitions. To achieve this, we create a Condensed Spanning Tree (CST) of the graph defined as follows:

The CST of a connected graph $G$ is a tree that includes all of the vertices and some of the edges of $G$. The CST is rooted. Every node has a unique parent node connected to it on the path to the root, and any number of children nodes. The CST structure has the following properties:

- Each CST node $u$, has a weight equal to $\left|S_{u}\right|$, where $S_{u}$ is the sub-tree that starts from node $u$. In weighted graphs the weight of $u$ is the sum of weights of nodes in $S_{u}$.

- The unique parent edge for each node $u$ in the CST has a weight equal to $\left|E_{u}\right|$, where $E_{u}$ includes all the 


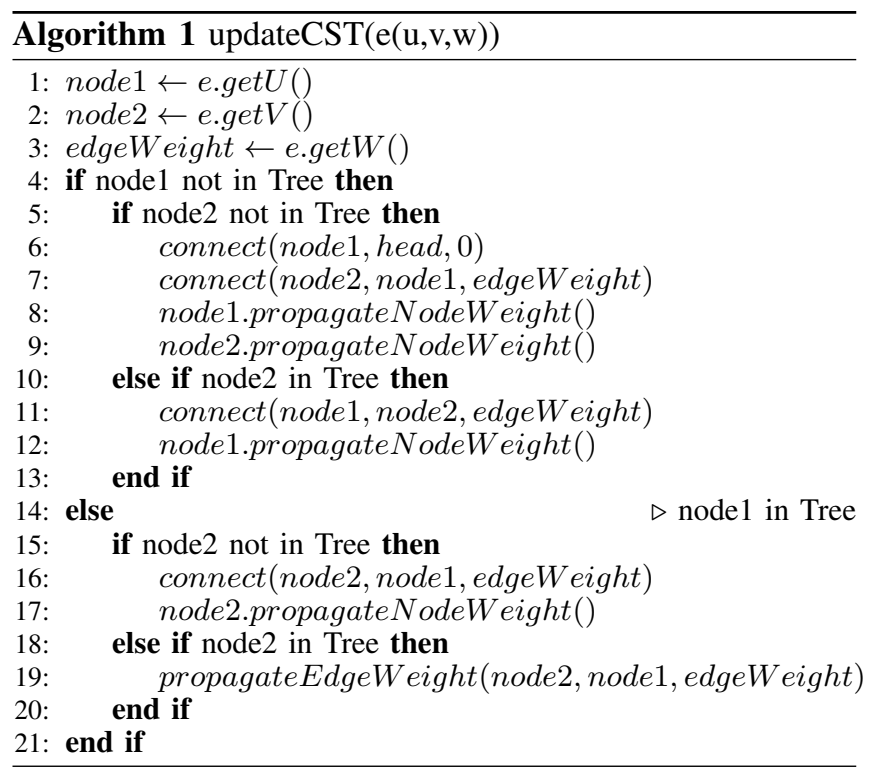

edges that start from a node in $S_{u}$ and end in another node $v \notin S_{u}$. In weighted graphs the parent edge of $u$ has a weight equal to the sum of weights of edges in $E_{u}$.

Thus, each node in the CST has a weight indicating the compound weight of its sub-tree. The weight of its parent edge indicates the number of edges that emanate from this sub-tree towards other nodes in the graph. Both statistics are utilized when forming the graph partitions.

\section{B. CST Creation Algorithm}

In the context of our streaming model where edges of a graph are arriving in arbitrary order, we form the CST by inserting nodes as they appear in the stream. Additional optimization's are discussed later in this subsection. First, in order to have the capability of creating a spanning tree for a graph that is not yet connected, we use as root a virtual node named as "head" with zero weight since this node will not affect the balance of the partitioning. Next, for every incoming edge $(u, v)$, our algorithm will place nodes in the CST structure using one of the following ways:

- If both nodes $u, v$ do not appear in the existing tree, the first node $u$ will be connected with the head node with edge weight zero (since this edge doesn't exist in the real graph and it will not affect the cut-edge). Then, node $v$ will be connected to $u$ with the provided edge weight.

- In cases where, from an incoming edge only one of its adjacent nodes exists in the CST, we connect the new node as a descendant to the node already present in the tree with the appropriate edge weight.

- If both incoming edge nodes have already been placed in the structure by previous insertions, we propagate the incoming edge weight to all existing edges in the unique path from node $u$ to $v$, as will be explained.
The CST is updated with every incoming edge $e(u, v, w(u, v))$ as described in Algorithm 1. After any node insertion in the tree, using the propagatedNodeWeight() function, we update the weights of certain CST nodes. Insertion of an edge between two nodes that already appear in the CST, triggers evaluation of the propagateEdgeWeight() function. Both propagation methods are described next:

Node weight Propagation: The node-weight propagation is a simple recursive function, and is called every time a new node is added to the tree structure. The inserted node adds its weight to its parent node, and this procedure continues recursively until all nodes up to the root of the tree are informed for the new node weight addition to the structure.

Edge weight Propagation: The edge-weight propagation is a technique to inform the CST about edges of the graph that are not present in the tree. For every such edge from node $u$ to node $v$ that both appear in the CST, we add its weight to every edge that participates in the path from $u$ to $v$ in CST. We know that in a tree, this path is unique. In order to quickly find the path between two nodes, we start the edge propagation with the node that has the biggest depth in the tree (counting from root to leaves). If the propagation procedure reaches the depth of the second node, but hasn't encounter it yet, then both nodes continue the propagation up to the root until they meet.

In order to illustrate the way we create a spanning tree from a streamed graph, we have formed an example in Figure 1. For simplicity we assume that all nodes and edges in the graph have weight equal to one. The order of the streamed edges is random, and for the described example is: $\{(\mathrm{a}, \mathrm{d})(\mathrm{a}, \mathrm{b})(\mathrm{c}, \mathrm{e})(\mathrm{c}, \mathrm{g})(\mathrm{d}, \mathrm{f})(\mathrm{g}, \mathrm{h})(\mathrm{a}, \mathrm{c})(\mathrm{b}, \mathrm{d})(\mathrm{e}, \mathrm{g})(\mathrm{c}, \mathrm{d})$ $(\mathrm{c}, \mathrm{h})(\mathrm{d}, \mathrm{h})(\mathrm{f}, \mathrm{h})\}$. In this example the number associated with every node in the created CST corresponds to the node weight calculated by the Node weight Propagation procedure. Moreover at every edge of the created CST we illustrate the total edge weight for each edge resulted after the whole stream is processed. The numbers in the arrows illustrate the total propagated edges form each sub-tree.

Our spanning tree creation algorithm makes no assumptions on the order of the incoming edges, since changes on a real growing graph are not predictable. Still, there are simple extensions to the algorithm that can improve performance. For instance, at system initialization, instead of processing streamed edges by their arrival order, we can buffer multiple edges and rearrange their order before inserting them in the CST, in a manner similar to bulk index creation. In our implementation, we utilize a simple process that constructs the initial CST by buffering incoming edges based on the available memory, sorting them in BFS order and then inserting them in the tree using Algorithm 1. The intuition behind the choice of BFS is that it preserves locality information in the stream [12].

\section{Creating, Monitoring And Adapting PARTITIONS}

Partitions in our framework are generated dynamically, while processing the graph stream. New nodes are placed 

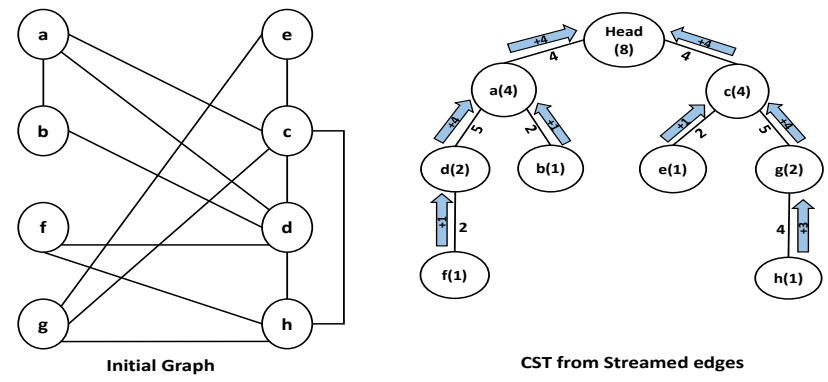

Figure 1. CST creation example from randomly streamed edges

in the appropriate partitions so as not to increase the edgecut and maintain balance. Details on this process are given in Subsection IV-A. In addition to creating the partitions dynamically, we may use the constructed CST in order to compute new partitions on demand, as is described in Subsection IV-B.

\section{A. Online creation of graph partitions}

In the unrestricted streaming model new edges of the graph in the form $((u, v), w(u, v))$ are presented sequentially in a stream. The constituent nodes $u$ and $v$ that define the edge, may have already appeared earlier in the stream or, in case this is the first occurrence of one or both of them, the new edge implies their insertion in the graph. In graph partitioning, when a node is placed in a partition, it maintains locally its adjacency list. Thus, each new edge augments the adjacency lists of its constituent nodes. Selection of the appropriate partition to place a new node is decided in real time using one of the following ways:

- If nodes $u, v$ first occur in the stream, they are both placed in the partition with the minimum total node weight. This way, we retain the balance of the partitions, as the graph evolves.

- In cases where, from an incoming edge only one of its adjacent nodes has been placed in a partition, then the new node is assigned in the same partition with its adjacent node, only if this insertion doesn't violate the balance criterion. Otherwise, the incoming node is placed in the partition with the minimum total weight.

The assignment of new nodes to partitions seeks to retain the locality of the partitions already computed by the system and, at the same time, guarantees that the desired balance criterion on the partitions weights is respected throughout the streaming process.

\section{B. Computing Graph Partitions On-demand}

We now describe how our techniques utilize the information stored in the CST so as to compute on-demand a new $k$ way partition of the graph seen thus far. Because this process has access to the accumulated history of the graph updates maintained by the CST, it often improves significantly the quality of the partitions, as our experiments demonstrate. Unlike one pass techniques that require re-streaming the whole graph, in our framework, we only utilize the CST in order to compute the partitions.

The proposed algorithm is based on an approximation greedy scheme [10] for the Multiple Knapsack problem (MKP), which is a natural generalization of the single knapsack problem. In MKP, we are given a set of $n$ items $a_{1}, \ldots a_{n}$ and $m$ bins, where each item has a cost $c(i)$ and a size $s(i)$. Each bin has a capacity $c(j)$, denoting the maximum total weight that a bin can hold. The goal in MKP is to find a subset of items of minimum cost such that they have a feasible packing in the bins. The greedy algorithm first sorts all items by the ratio of their costs to their sizes such that $\frac{c(1)}{s(1)} \leq \frac{c(2)}{s(2)} \leq \cdots \leq \frac{c(n)}{s(n)}$ and then it greedily adds items to bins (one bin at a time) until it finds an item $a_{i}$ that exceeds the bin capacity. The algorithm chooses the minimum cost solution between $\left\{a_{1}, a_{2}, \ldots, a_{i-1}\right\}$ and $\left\{a_{i}\right\}$ for each bin.

In our work, we transform the graph partitioning problem into a MKP instance by considering as "items" all nodes in the CST along with their subtrees (recall that a CST node is also a graph node). For every node $u$ in the CST, we assign as $c(u)=$ parent $E d g e W$ eight $(u)$, which suggests that by choosing a sub-tree starting from $u$ and placing it to a partition, we will pay the corresponding cost to the edge cut of the solution. Also we denote as $s(u)=$ weight $(u)$, where weight $(u)$ indicates the total number of nodes in the subtree starting from $u$. Beside that, the $k$ partitions that we want to divide the nodes can be seen as the bins where the capacity for each bin $c(j)$ denotes the maximum weight a partition can hold, in our case $c(j)=(1+\epsilon)\lceil|V| / k\rceil$. The goal of the algorithm remains the same, that is, find a feasible balance partitioning of the sub-trees with the minimum cost.

Just like the MKP, our algorithm sorts nodes by their amortized cost $\frac{\text { parentEdgeWeight }(u)}{\text { weight }(u)}$ in ascending order. The intuition is that we would seek to add to a partition a group of nodes (the sub-tree of $u$ ) that results in a small number of cut edges, when the later is amortized over the size of the group (larger groups are expected to induce larger edge cuts). Next, the algorithm repeatably selects nodes from the list that do not violate the balance (bin capacity) criterion and place their entire sub-tree to the current forming partition. When $k$-1 partitions have been formed, the algorithm assigns the remaining nodes to the last, $k^{t h}$ partition.

In this process, two small modifications to the MKP greedy algorithm are required in order to meet our goal parameters. First, unlike MKP we try to fill each partition above a minimum load threshold since leaving half-empty partitions will result in overloading others. Thus, at the beginning of the partitioning procedure, the maximum and the minimum load for each partition are calculated. The value of the minimum load is recalculated, after a new partition is formed (a bin is filled up with items), in order to properly divide the remaining nodes to the other partitions and not to overload any specific part. When the algorithm reaches a node that cannot fit in the current partition it will try to find the next node in the list with the appropriate 
weight so as to fill the partition up to the minimum required weight.

The second modification of our algorithm is that during its execution, the weights of the nodes are being adjusted, when nodes are placed inside a partition. Initially, the weight of a node equals its weight in the CST. In the process, this value is adjusted in order to reflect the current number of nodes remaining in the sub-tree starting from this node after any subtraction of sub-trees during the partitioning procedure. This is achieved via an unpropagateWeight() function that every time a sub-tree is selected and assigned to a partition subtracts the whole sub-tree weight from all nodes up to the head of the tree.

The greedy algorithm does not attempt to update the amortized cost of a node, while the partitions are formed. When a sub-tree underneath a node $u$ is chosen and that part is removed from the sub-tree of $u$, the exact reduction in the edge cut value for $u$ and the corresponding update of its cost can not be determined from the CST, as this would require access to the full graph. By not updating the edge-cut estimate, we essentially assume that removing a part of the sub-tree of $u$ does not alter its "average" connectivity to the rest of the graph based on the remaining nodes. We have also experimented with heuristic approaches for updating the edge weights but observed only marginal differences to the quality of the described algorithm. Moreover, by not updating the estimated costs, the greedy algorithm requires a single sorting of the initial list of nodes. Thus, the running time of the presented algorithm is $O(n \times \log n+k \times h \times n)$, where $n$ is the number of nodes in the CST and $h$ its height.

A benefit of our technique is that we can utilize the statistics of the CST and compute an upper-bound of the edge-cut of a partitioning scheme (given the number of partitions $k$ ) by summing up the edge weights of the selected subtrees in a dry-run of the algorithm. Thus, the application may choose $k$ accordingly and then perform the actual partitioning process.

For a working example of the way the greedy algorithm performs we will use the CST of Figure 1. In Figure 2 we illustrate the CST structure with the appropriate weights on nodes and edges. We form the amortized cost list for every node in the CST which is 1 for nodes $\{a, c\}, 2$ for nodes $\{b, e, f\}, 2.5$ for nodes $\{d, g\}$ and 4 for node $h$. We first sort the nodes in increasing order of their amortized costs: $a \leq c \leq b \leq e \leq f \leq d \leq g \leq h$. For performing partitioning for $k=2$, our algorithm will first choose the subtree starting from node $a$ (nodes $\{a, b, d, f\}$ ), since it is first in the amortized cost list and its weight (4) doesn't exceed the maximum load of the partition. Next, it will form the second partition with the remaining nodes $\{c, e, g, h\}$.

Trying to form a partition for $k=3$ in the same example we need every partition to have at least 2 and maximum 3 nodes. Looking at the sorted list while forming the first partition we cannot select node $a$ nor $c$ since they both have a weight (4) and doesn't fit into a bin (partition). Thus, we move to the next items in the list and we place nodes $\{b, e\}$

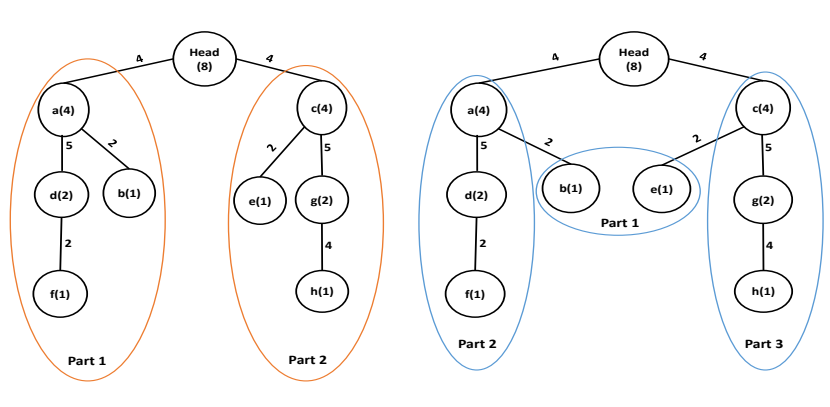

Figure 2. CST partitioning example for $\mathrm{k}=2$ and $\mathrm{k}=3$

in the first partition. For the second partition, we now can select node $a$, since its weight is now 3 (decreased by 1 from the removal of node $b$ in the first iteration) and place the remaining nodes in the sub-tree of $a$ to the second partition (nodes $\{a, d, f\}$ ). Last we form the third partition with the remaining nodes $\{c, g, h\}$.

When the algorithm is used for re-partitioning a graph, the application may wish to also account for the cost of migrating nodes between partitions. In such cases, the cost may be computed as:

$$
\text { migrationCost }(u)=c(u) \times M(u)^{-1}
$$

where $M(u)$ is a positive function that decreases the cost of nodes when, while re-partitioning, node $u$ is assigned to the same partition as before. In our implementation we use a simple function to favor solutions with small migration overhead:

$M(u)= \begin{cases}1+m & \text { if oldPartition(u) }=\text { newPartition(u) } \\ 1 & \text {,otherwise }\end{cases}$

Parameter $m>0$ is used to steer solutions towards new partitions with small migration cost. Of course, other functions can be used as well.

\section{Controlling the Size of THe CST}

In this section we describe an intuitive technique that dynamically compresses the CST structure when processing the stream. Compression is achieved by creating supernodes that replace multiple nodes of the original CST, thus reducing its size. This grouping is performed on-line, i.e. when processing the incoming stream, by making small adjustments to the updateCST() function (Algorithm 1). As a result, the dynamic compression process has no impact in the rate in which incoming tuples are processed. In fact, processing new tuples using the compressed CST is faster because of its smaller size. Moreover, because creation of the super-nodes in the modified CST structure is performed in a manner that preservers node locality, the resulting compressed CST manages to produce partitions of similar quality than the uncompressed CST, as will be evident by our experiments.

The modified CST structure is a tree of super-nodes. Each super-node has an id (name) $u$ that corresponds to a node in the graph and contains a non-empty set of graph nodes 

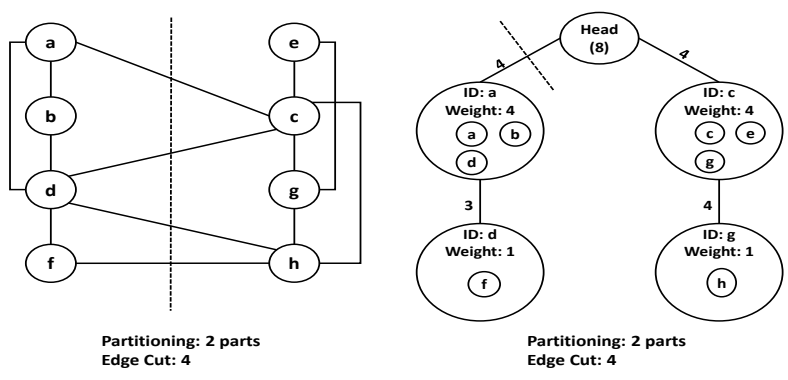

Figure 3. Compressed CST for running example

$v_{1}, v_{2}, \ldots$ If node $v_{i}$ is placed inside a super-node with id $u$, then one of the following conditions holds: either $v_{i}=u$, or $v_{i}$ is a neighbor of $u$. The weight of the super-node equals to the weight of all nodes $v_{i}$ placed inside it. We say that node $v$ exists in the tree, if $v$ appears inside one of the supernodes. As will be evident by its construction, node $v$ may exist in no-more than one super-node in the CST. Creation and maintenance of the super-nodes requires the following changes to the CST update algorithm when processing a streamed edge $(u, v, w(u, v))$ :

- If neither node $u$, nor $v$ exist in the tree, then a new super-node with id $u$ will be formed and both nodes will be placed inside it. The super-node will be connected to the virtual head node with edge weight zero. The supernode total weight will be propagated to the root. By creating a super-node with id that of $u$, we essentially create a placeholder where all neighbors of node $u$ (in addition to node $v$ ) that may appear later in the stream, will be accumulated on.

- If both nodes $u, v$ already exist in the tree, only the edge weight will be propagated to the unique path between the corresponding super-nodes that contain $u$ and $v$ in the tree structure. In the trivial case when both $u$ and $v$ are stored in the same super-node, then no edge weight propagation is performed.

- If only one of the nodes (for instance $u$ ) appears in the tree, we distinguish the following two cases:

1) If there is a super-node with $\mathrm{id}=u$ previously created, we place its newly discovered neighbor $v$ inside that super-node. We also increase the supernode weight by 1 (because of the addition of $v$ to the super-node) and propagate the new super-node weight to the head (informing the structure for the addition of the node).

2) If the previous condition doesn't hold, then there is a super-node with id $w$ that contains $u$ (i.e. $u$ has been observed as a neighbor of node $w$ in the past). In that case, we create a new supernode with the id of $u$ and place $v$ inside the newly created super-node. Notice that $u$ is not placed inside the new super-node with id $u$, as $u$ is already placed in the super-node with id $w$. The new super-node is added as a child of $w$ in the CST, in order to preserve locality: $v$ is a neighbor of $u$, which is a neighbor of $w$, and are thus stored in two super-nodes that have a parentchild relationship in the CST. Since node $u$ existed already in the CST, only the node weight of $v$ needs to be propagated.

In the example of Figure 3, we illustrate the compressed CST creation algorithm in the same example used in Figure 1. Again for simplicity we assume that all nodes and edges in the graph have weight equal to one. The same stream order is assumed: $\{(\mathrm{a}, \mathrm{d})(\mathrm{a}, \mathrm{b})(\mathrm{c}, \mathrm{e})(\mathrm{c}, \mathrm{g})(\mathrm{d}, \mathrm{f})(\mathrm{g}, \mathrm{h})(\mathrm{a}, \mathrm{c})(\mathrm{b}, \mathrm{d})$ $(\mathrm{e}, \mathrm{g})(\mathrm{c}, \mathrm{d})(\mathrm{c}, \mathrm{h})(\mathrm{d}, \mathrm{h})(\mathrm{f}, \mathrm{h})\}$. At the beginning of the stream the virtual node is inserted in the tree, and edge $(a, d)$ is to be added. Neither of these nodes exist in the CST, and a super-node with $\mathrm{id}=a$ is created, with both nodes $a$ and $d$ placed inside it. Next, edge $(a, b)$ is streamed, where node $a$ already exists in super-node $a$. Thus, node $b$ is also placed inside that super-node. The same procedure continues for edges $(c, e)$ and $(c, g)$. When edge $(d, f)$ arrives, we find node $d$ already present in the tree but in a super-node with $\mathrm{id}=a$ and there is no super-node with $\mathrm{id}=d$. Notice that, by construction, if such super-node existed, it would have been a child of super-node $a$, where $d$ is placed. We, thus, form a new super-node $d$ and place $f$ inside it. This procedure continues until the end of the stream.

Overall the new CST construction procedure preserves node locality. All neighbors of a node $u$ are either placed in the same super-node with $u$ (case of nodes $a, c$ in the figure), or in a super-node with distance only one from the one that contains $u$ (e.g. case of nodes $d$ and $g$ in the running example). Regarding the space-reduction achieved, we notice that in the end only 5 nodes have been created (4 super-nodes and the head) instead of 9 nodes that the uncompressed CST contains (Figure 1) and, respectively, the number of edges has been reduced from 8 to 4 . The space-savings stem from the fewer edges that are present in the compressed CST, because of the fewer number of supernodes compared to the number of nodes in the uncompressed structure. The number of nodes stored in the compressed CST is equal to the number of nodes in the original CST, thus there is no space savings there. The depicted super-node ids may be omitted in the implementation because either the super node with id $u$ contains $u$ (e.g. nodes $a, c$ in the figure) and there is no reason to store both descriptors, or node $u$ is placed in the parent of the super node (e.g. super-node $g$ can be linked directly to node $g$ inside super-node $c$ and thus its id may be inferred. As a result, in a more compact representation, one doesn't need to store the ids of the supernodes.

\section{EXPERIMENTS}

In this section we present experimental results of our framework against alternative methods. We first describe our experimental setup in Section VI-A and then present an evaluation of our suggested online and on demand partitioning methods for unrestricted graph streams in Section VI-B. We show results on different application scenarios that 


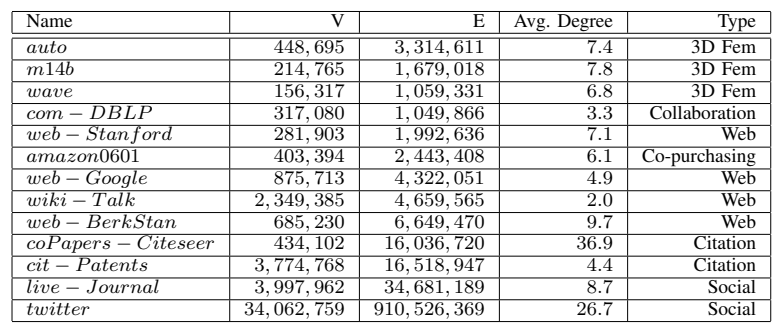

Table I

SUMMARY OF THE EVALUATION DATASETS

our framework can accommodate with respect to adapting the partitions dynamically based on quality criteria or the desired elasticity of the partitioning scheme. In Section VI-C we present an evaluation of the compressed CST structure and showcase the ability to significantly reduce its size and, at the same time, maintain the ability to compute high quality partitions. Finally, in Section VI-D we evaluate the stability of our method when graph streams of different random orders are processed.

\section{A. Experimental Setup}

For our evaluation we use a diverse collection of synthetic and real-world graphs with various sizes and different edge distributions. Table I summarizes the basic statistics about each graph used in our experiments, as well as its type. The datasets were chosen to balance both size and variety and are frequently used in evaluating graph partitioning algorithms [8]. All algorithms proposed in this paper have been implemented in JAVA, and all experiments were performed in a single machine, with Intel Core CPU i7-4700MQ, and $8 \mathrm{~GB}$ of main memory.

To the best of our knowledge, the only alternative technique that can be used in the case of unrestricted graph streams that we consider in this work is hash-based partitioning where the assignment of each node to a partition is computed via a hash function over its id. This scheme is utilized by many popular graph processing platforms. In addition in our experimental evaluation we have adapted two on-line partitioning algorithms, Linear Deterministic Greedy (LDG) [15] and FENNEL [16] in order to compare them with our techniques. Linear Deterministic Greedy (LDG), place a newly arrived node $u$ to the partition $V_{i}$ that maximizes $\left|N(u) \cap V_{i}\right| \times\left(1-\frac{\left|V_{i}\right|}{n / k}\right)$ and FENNEL to the partition $V_{i}$ that maximizes $\left|N(u) \cap V_{i}\right|-\alpha \frac{\gamma}{2}\left|V_{i}\right|^{\gamma-1}$. Where $N(u)$ denotes the number of neighbors of node $u$ (incoming with each node in the stream).

In our evaluation, we measure the edge-cut ratio $\lambda$ defined as the number of cut-edges of the resulting partitioning scheme to the total number of edges appeared in the graph stream at the time the measurement was taken. In all experiments we assume a default value of 0.05 for the imbalance parameter $\epsilon$ discussed in Section II. Unless otherwise specified, the number of partitions used was $k=16$.

\section{B. Online and On-demand Partitioning on Unrestricted Graph Streams}

We here present experiments of our online and on-demand partitioning methods in the suggested unrestricted graph stream model, where edges of the graph are presented in random order. In these experiments we used a 30\% of the graph edges for initialization and present results for streaming the remaining part of the graphs in random order. The same technique is used in the comparison with the online algorithms, creating partial adjacency lists for every node based on the percentage of the graph that have already been streamed. When processing the graph stream, we have implemented both the unbuffered and buffered option for updating the CST. In the unbuffered execution, the edges are processed in the order of appearance in the stream. In the buffered execution, a buffer that can hold up to $10 \%$ of the edges is used. Edges are accumulated in that buffer and are ordered by the ids of their starting, ending nodes before being processed (one-at-a-time) by the CST.

In Figure 4, we compare our proposed method to current one-pass partitioning algorithms for various datasets and for a default value of $k=16$. Moreover we use hash partitioning as an upper bound because this approach completely ignores the edges and the locality of the graph. We present the values of $\lambda$ after the whole graph is processed by each algorithm. We notice that in all settings, our technique produces partitions that reduce the edge-cut independently of the graph average degree and volume, in comparison to the one-pass algorithms.

In Figure 5 we present results for our adaptive streaming model in Web BerkStan dataset. We mention that the behavior of our algorithm is presented in some specific datasets due to space limitations, but was tested in all the experimental datasets with similar results. The CST line correspond to the unbuffered execution of our online creation algorithm of Section IV-A. We also evaluate the unbuffered algorithm when the partitions are being adapted whenever a $10 \%$ increment of the graph has been processed. Adapting the partitions is done using the algorithm of Section IV-B. We further include results of partition adaptation in the buffered execution. Finally, as a baseline, we also depict performance of Hash partitioning. We observe that our techniques significantly outperform Hash partitioning. The periodic adaptation of the partitions from the CST helps improve performance further. Additionally, buffered execution seems to provide significant gain in this experiment.

In Figure 6 we assume that an upper threshold on the value of $\lambda$ is specified by the application. Whenever this limit is exceeded, partitions are being adapted, as is shown in the figure. We observe that our techniques can easily repartition the graph, to retain or even improve the edge-cut. In addition to adapting the partitions based on an edge-cut limit, our techniques permit us to dynamically adjust their number, based on the application's needs.

Last in Figure 7, we present results of adapting partitions on-demand, using the migration gain function described in 


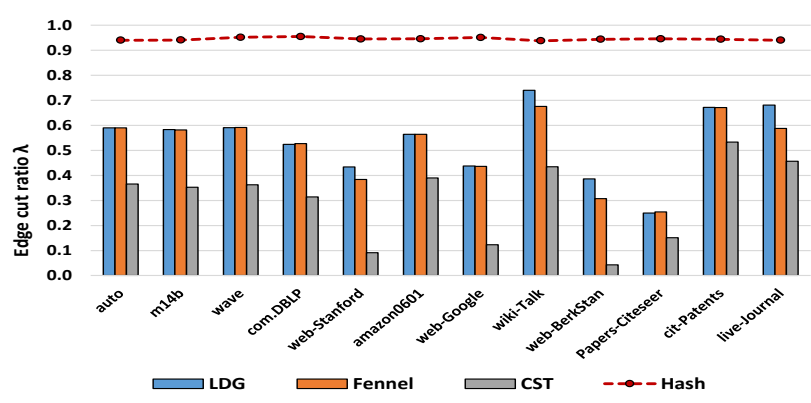

Figure 4. Comparison to Online Algorithms

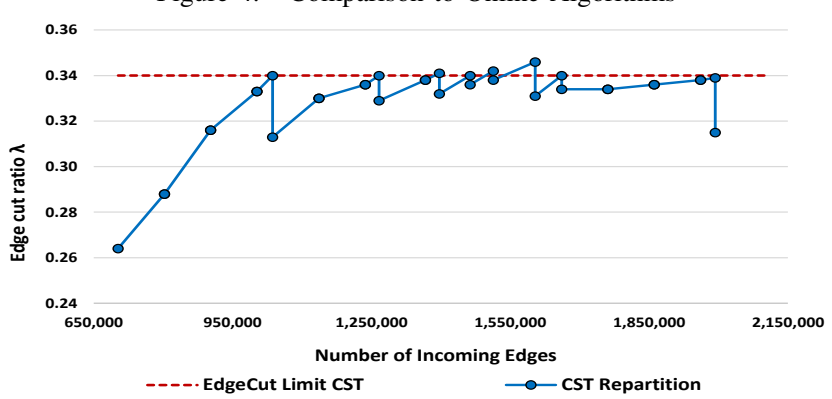

Figure 6. Adaptive CST Partitioning with edge-cut limit for web-Stanford graph

Section IV-B. Specifically we vary the migration penalty from 0.25 to 32 , and we measure the corresponding edgecut and node migration. We notice that for $m=32$ (final point), the migration between partitions has been minimized ( $4.1 \%$ of the nodes), but still the edge-cut remains about the same $(\lambda=0.375)$ as the one that doesn't consider the migration at all $(\lambda=0.335)$.

\section{Compressed CST Evaluation}

In Figures 8 and 9 we evaluate the edge-cut quality and the compression ratio of various CST alternatives. The edgecut for each graph was measured only once at the end of the stream (no intermediate re-partitions). The on-demand compression results presented in the figures refer to an alternative compression method that reduces the CST size when a maximum space limit is reached. The technique compacts whole sub-trees to super-nodes with weight equal to the total number of nodes in the sub-tree. The super-node obtains the same parent edge as the sub-tree. The weight of this edge represents the out-going edges from this sub-tree to others. The compression procedure selects sub-trees in increasing order based on their amortized costs, as defined in Subsection IV-B.

In Figure 8, we observe that the edge-cut values resulted from the use of the CST and the compressed CST are about the same, even though the corresponding size reduction is often significant, as seen in Figure 9. In the later, we depict the ratio of the size of the corresponding CST structure over the size of the graph, both measured in binary format.

In Figure 10 we depict the execution times of the partitiong algorithm of Section IV-B. Using the CST resulted
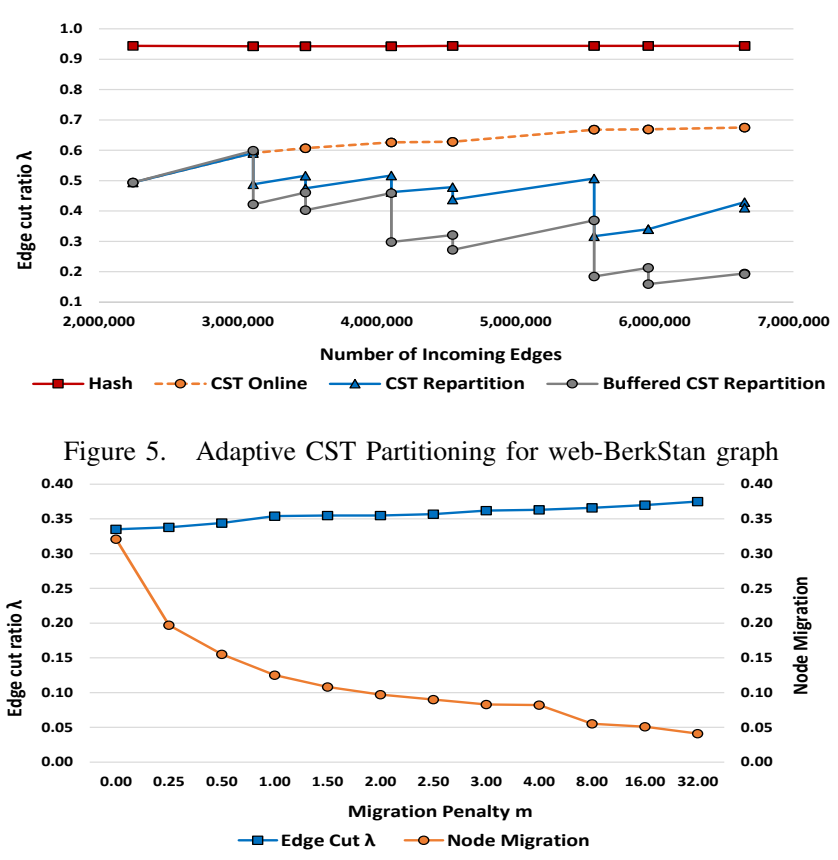

Figure 7. CST Partitioning with various values of $\mathrm{m}$ for web-Stanford graph $(k=16)$

from the on-demand compression, the algorithm runs in less than one second, for most of the graphs.

In Table II we present the edge-cut ratio $(\lambda)$ for the twitter dataset, which is the largest graph in our dataset collection. Twitter social graph is a directed graph with every edge expressing a follower relationship between two users. The provided results are calculated on the unbuffered compressed CST which is about $3.5 \%$ of the twitter graph size. The on-demand compressed CST takes $2 \%$ of the graph and computes partitions on average time of $135 \mathrm{sec}$. Moreover, the CST produces partitions that significantly reduce the edge-cut for all different values of $k$, compared to the online algorithms.

\begin{tabular}{|c|l|l|l|l|}
\hline Number of parts (k) & CST & LDG & FENNEL & HASH \\
\hline 2 & 0.151 & 0.349 & 0.351 & 0.498 \\
\hline 4 & 0.381 & 0.525 & 0.493 & 0.749 \\
\hline 8 & 0.467 & 0.611 & 0.591 & 0.875 \\
\hline 16 & 0.680 & 0.732 & 0.712 & 0.937 \\
\hline \multicolumn{4}{|c}{ Table II }
\end{tabular}

EDGE-CUt FOR THE TWITTER Social GRAPH

In Figure 11 we measure the rate at which the different versions of the CST can process the graph stream and assign nodes to partitions. These calculations include the time to process a stream tuple after it has appeared in the stream buffer, assign its nodes to partitions and perform any required modifications in the CST (addition of nodes, node/edge weight propagation). We started with an empty tree and processed continuously the entire graph (16 Million edges). We smooth the depicted lines by taking average measurements whenever a $10 \%$ of the stream is processed. We notice that even the unbuffered execution enables us 


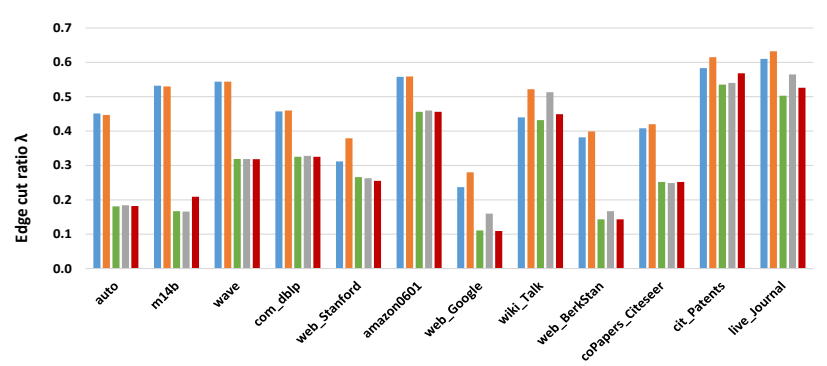

$\varpi$ CST $₫$ Compressed CST $\backsim$ Buffered CST $\llbracket$ Compressed Buffered CST $\backsim$ OnDemand Compression

Figure 8. Edge Cut for CST and Compressed CST

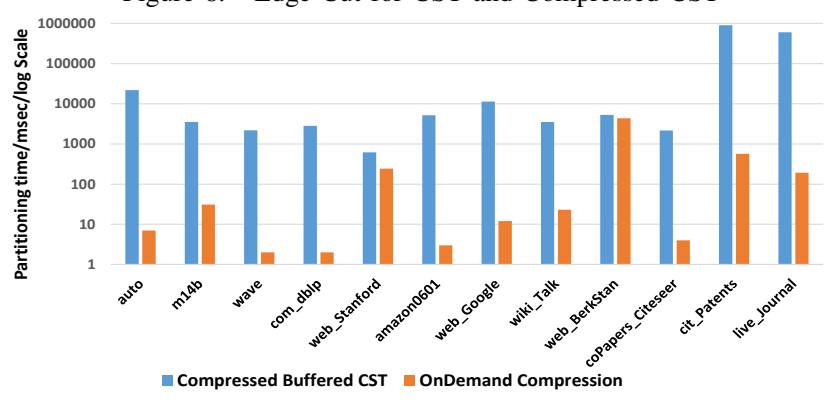

Figure 10. Partitioning Time

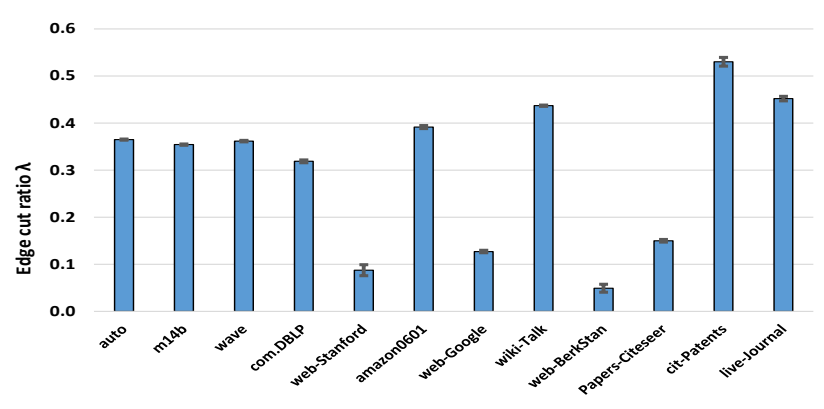

Figure 12. Sensitivity to Stream Order

to process about 1 Million stream edges per second. The compressed CST is slightly faster, because of its smaller size. Buffered execution, helps increase rate even-further, topping at 4.7 Million edges per second.

\section{Stability Evaluation}

In Figure 12 we provide an experimental evaluation of the performance of our on-demand partitioning algorithm in different CST structures produced by different random orders for the unrestricted stream model. In particular, for each graph presented in the figure we depict the average edge-cut from 100 different random orders of unrestricted streams using their corresponding CSTs produced by the plain algorithm (no bulk-loading, buffering nor compression were used). In addition, we depict using error bars the standard deviation of the computed edge-cuts.

We notice that for all datasets the differences between the runs are negligible, as highlighted by the error bars in the figure. This happens because in all CSTs, a partial locality of the nodes is preserved due to the way the tree is constructed (Section III-B). Even in cases of bad

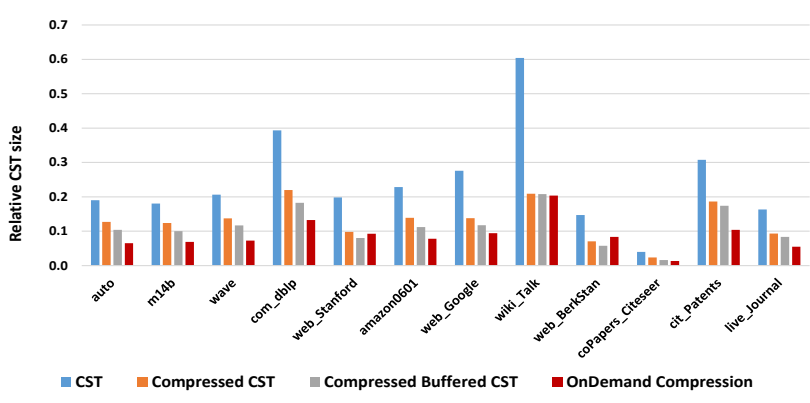

Figure 9. Compression Ratio of CST Structures

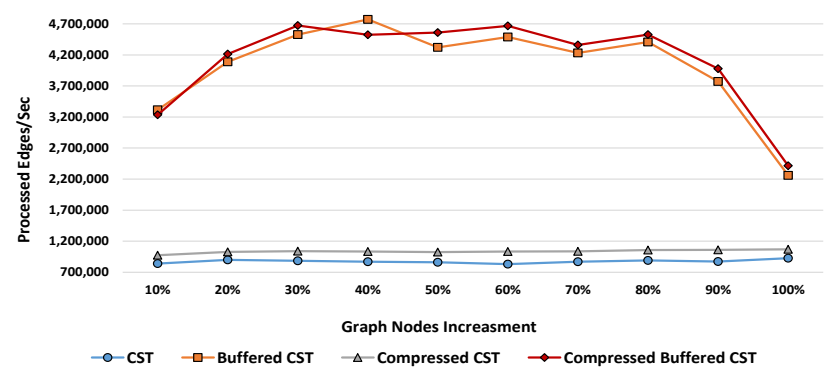

Figure 11. Stream processing rate, coPapersCiteseer dataset

orderings (trees that induce long edge propagation for some nodes) the partitioning algorithm itself will avoid selecting the affected sub-trees, because their amortized cost will be higher (Section IV-B).

As described in Section III-B, using the CST we can compute an upper-bound of the edge-cut, without having to perform the actual partition. An extension that we leave as feature work is to select sub-trees of the CST and re-stream their edges, so as to reposition them in the tree and compute paritions of even-higher quality.

\section{RELATED WORK}

Graph partitioning is a well studied NP-hard problem [3]. The static balanced graph partitioning algorithms (offline) remains NP even in the case of $k=2$ (bisection) and also if one relaxes the balanced constraint [1]. There are many approximation algorithms for this problem. Also there are many offline heuristics of different nature, spectral [2], geometric [5], combinatorial [7] and multilevel [6] that also solve this problem but with no performance guarantees. An aggressive replication-based approach is also suggested in [11] that permits replication of nodes in order to minimize the number of distributed traversals. In practice, all the above heuristics are quite effective, and many tools are available, like Chaco [4] and METIS [6]. However, because of their static nature they are not best suited for big and evolving graphs.

On the other hand, one-pass (online) streaming algorithms recently introduced [12], [15], [16], consider the graph partitioning problem in the restricted streaming model where graph nodes are loaded continuously in a stream, each with its complete adjacency list. Online algorithms mainly address the problem of partitioning graphs that can 
not physically fit in memory and, therefore, static methods cannot be used. The quality of the produced partitions mostly depends on the stream order of the incoming nodes but also on the objective function that each algorithm uses and is significantly lower to static partitioners. The restricted streaming model used by one-pass algorithms cannot adapt in situations where the graph evolves over time. Repartitioning the graph requires that the whole streaming process is performed from scratch leading most of the times to increased node movement between parts. Also online techniques cannot adapt to the case where the number of parts is not initially fixed and will be modified at run-time. Finally, another drawback of online approaches is that their objective functions are not adapted to weighted graphs.

A separate field on graph partitioning studies the problem of re-partitioning algorithms where the objectives are to minimize the node migration from the old partition to the new one but also to reduce the time required for calculating the new partitions. There are many works in the field of re-partitioning algorithms. Nowadays, the most common approaches used are: scratch-remap [13], diffusion [14], or biased partitioning with fixed vertices. All re-partitioning algorithms are static approaches, in the sense that they all require the whole graph structure and global data computations are involved in producing a new partition.

To the best of our knowledge this work first addressed the partitioning problem combining all the characteristics of the three main partitioning methods described above (offline, online and repartitioning). Our framework can be easily used as a replacement for these approaches in cases of big and evolving graphs that dynamically change. Our approach produces fast high quality partitions to any type of graphs, doesn't require as input the whole graph but assigns nodes dynamically to partitions and can adapt to changes by repartitioning the graph at any given time with varying number of parts and balance restrictions further taking the migration cost into account.

\section{CONCLUSIONS}

In this paper we first presented a graph steam model that, compared to the model used in prior graph partitioning algorithms, is more generic and is, thus, applicable to modern applications that manipulate large graphs that constantly evolve. We then introduced an intuitive summary spanning tree structure, the CST, which is significantly smaller that the evolving graph, can be maintained in real time while the graph stream is processed, and, more importantly, retains intuitive statistics so that a high-quality partitioning scheme can be computed from it. We presented efficient algorithms for creating the CST and computing graph partitions using its information, either online or on-demand. We also discussed several extensions to the basic structure that help improve performance or can be used to restrict its size to better fit the available memory constraints of the system.

Our experimental evaluation demonstrated that our framework produces better quality partitions than prior techniques.
The proposed algorithms have low time and space complexity and, as our experiments demonstrate, can be implemented using a common PC for processing millions of graph edges per second. At the same time, they can be adapted to accommodate different needs of the application with respect to the node migration cost, the quality of the produced partitions or their maximum load.

\section{ACKNOWLEDGEMENTS}

This research has been co-financed by the European Union (European Social Fund ESF) and Greek national funds through the Operational Program "Education and Lifelong Learning" of the National Strategic Reference Framework (NSRF) - Research Funding Program: RECOST.

\section{REFERENCES}

[1] K. Andreev and H. Räcke. Balanced Graph Partitioning. In Proc. of SPAA, pages 120-124, New York, NY, USA, 2004.

[2] M. Fiedler. A property of eigenvectors of nonnegative symmetric matrices and its application to graph theory. Czechoslovak Mathematical Journal, 25(4):619-633, 1975.

[3] M. R. Garey, D. S. Johnson, and L. Stockmeyer. Some simplified np-complete problems. In Proceedings of STOC, pages 47-63, New York, NY, USA, 1974.

[4] B. Hendrickson and R. Leland. A multilevel algorithm for partitioning graphs. In Proceedings of Supercomputing, New York, NY, USA, 1995.

[5] H. D. S. I, H. D. Simon, and H. D. Simon. Partitioning of unstructured problems for parallel processing, 1991.

[6] G. Karypis and V. Kumar. Multilevel k-way partitioning scheme for irregular graphs. J. Parallel Distrib. Comput., 48(1):96-129, Jan. 1998.

[7] B. Kernighan and S. Lin. An Efficient Heuristic Procedure for Partitioning Graphs. The Bell Systems Technical Journal, 49(2), 1970.

[8] J. Leskovec and A. Krevl. SNAP Datasets: Stanford large network dataset collection, June 2014.

[9] Y. Low, J. Gonzalez, A. Kyrola, D. Bickson, C. Guestrin, and J. M. Hellerstein. Graphlab: A New Parallel Framework for Machine Learning. In Proc. of UAI, July 2010.

[10] S. Martello and P. Toth. Knapsack Problems: Algorithms and Computer Implementations. John Wiley \& Sons, Inc., New York, NY, USA, 1990.

[11] J. Mondal and A. Deshpande. Managing large dynamic graphs efficiently. In Proceedings of SIGMOD, pages 145156, New York, NY, USA, 2012. ACM.

[12] J. Nishimura and J. Ugander. Restreaming graph partitioning: Simple versatile algorithms for advanced balancing. In Proc. of SIGKDD, pages 1106-1114, New York, NY, USA.

[13] L. Oliker and R. Biswas. Plum: Parallel load balancing for adaptive unstructured meshes, 1998.

[14] K. Schloegel, G. Karypis, and V. Kumar. Wavefront diffusion and lmsr: Algorithms for dynamic repartitioning of adaptive meshes. IEEE Trans. Par. Distr. Syst., 12(5):451-466, 2001.

[15] I. Stanton and G. Kliot. Streaming graph partitioning for large distributed graphs. In Proceedings of SIGKDD, pages 1222-1230, New York, NY, USA, 2012.

[16] C. Tsourakakis, C. Gkantsidis, B. Radunovic, and M. Vojnovic. Fennel: Streaming graph partitioning for massive scale graphs. In Proceedings of WSDM, pages 333-342, New York, NY, USA, 2014. 